\title{
POINTWISE AND NORM CONVERGENCE OF A CLASS OF BIORTHOGONAL EXPANSIONS
}

\author{
BY
}

HAROLD E. BENZINGER

\begin{abstract}
Let $\left\{u_{k}(x)\right\},\left\{v_{k}(x)\right\}, k=0, \pm 1, \ldots, 0<x<1$, be sequences of functions in $L^{\infty}(0,1)$, such that $\left(u_{k}, v_{j}\right)=\delta_{k j}$. Let $\phi_{k}(x)=\exp 2 k \pi i x$. It is shown that if for a given $p, 1<p<\infty$, the sequence $\left\{u_{k}\right\}$ is complete in $L^{p}(0,1)$, and $\left\{v_{k}\right\}$ is complete in $L^{q}(0,1), p q=p+q$, and if the $u_{k}$ 's, $v_{j}$ 's are asymptotically related to the $\phi_{k}$ 's, in a sense to be made precise, then $\left\{u_{k}\right\}$ is a basis for $L^{p}(0,1)$, equivalent to the basis $\left\{\phi_{k}\right\}$, and for every $f$ in $L^{p}(0,1)$, $\Sigma_{-\infty}^{\infty}\left(f, v_{k}\right) u_{k}(x)=f(x)$ a.e. This result is then applied to the eigenfunction expansions of a large class of ordinary differential operators.
\end{abstract}

1. Introduction. Let $\tau$ denote the $n$th order linear differential expression defined for suitable functions $u$ by

$$
\tau(u)=u^{(n)}+a_{n-2}(x) u^{(n-2)}+\cdots+a_{0}(x) u
$$

for $0<x<1$, where $a_{j}(x)$ is in $L^{\infty}(0,1), j=0, \ldots, n-2$. (When $n=1$, $\tau u=u^{(1)}$.) Let $U_{1}, \ldots, U_{n}$ denote $n$ linearly independent linear forms in $u$ and its first $n-1$ derivatives, evaluated at 0 and at 1 . Without loss of generality, we can assume that the $U_{j}^{\prime}$ 's are in normalized form $[18, \mathrm{p} .48]$ :

$$
U_{j} u=\alpha_{j} u^{\left(k_{j}\right)}(0)+\beta_{j} u^{\left(k_{j}\right)}(1)+\sum_{k=0}^{k_{j}-1}\left[\alpha_{j k} u^{(k)}(0)+\beta_{j k} u^{(k)}(1)\right],
$$

where $j=1, \ldots, n, 0 \leqslant k_{j} \leqslant n-1, k_{j+1} \leqslant k_{j}, k_{j+2}<k_{j},\left|\alpha_{j}\right|+\left|\beta_{j}\right|>0$. The set $U=\left(U_{1}, \ldots, U_{n}\right)$ is Birkhoff regular [18, p. 49] if certain determinants involving the $\alpha_{j}$ 's, $\beta_{j}$ 's, $k_{j}$ 's are not zero. The pair $(\tau, U)$ denotes the problem of solving

$$
\tau(u)=\lambda u,
$$

where $\lambda$ is a complex parameter, subject to the boundary condition

$$
U u=0 .
$$

It is assumed that $u$ is in $C^{n-1}[0,1]$, and that $u^{(n-1)}$ is absolutely continuous on $[0,1]$.

In [3] it was proved that if $U$ is Birkhoff regular, then the system of

Received by the editors June 18, 1975.

AMS (MOS) subject classifications (1970). Primary 42A60; Secondary 34B25.

Key words and phrases. Biorthogonal expansions, maximal function mapping, eigenfunction expansions. 
eigenfunctions and generalized eigenfunctions of $(\tau, U)$ is a (Schauder) basis for $L^{p}(0,1), 1<p<\infty$.

Definition. The problem $(\tau, U)$ has simple spectrum if $n$ is odd, or if $n$ is even and $\theta_{0}^{2}-4 \theta_{1} \theta_{-1} \neq 0$. (See [18, p. 49] for notation.)

In $\S 5$ we shall derive new asymptotic estimates for the root functions $\left\{u_{k}\right\}$ of a Birkhoff regular problem with simple spectrum, and for the root functions $\left\{v_{k}\right\}$ of the adjoint problem. Consequently, these biorthogonal systems have the properties stated in the abstract.

In [3] it was proved that the system of eigenfunctions of any Birkhoff regular problem is a basis for $L^{p}(0,1), 1<p<\infty$. The result provided no information on the problem of determining if such bases are unconditional. In the case that $p=2$, this question was considered by Dunford and Schwartz, who proved that any differential operator in $L^{2}(0,1)$ generated by a Birkhoff regular problem with simple spectrum is a spectral operator, and consequently the root functions are an unconditional basis for $L^{2}(0,1)$. Since the trigonometric system $\left\{\phi_{k}\right\}$ is not unconditional when $p \neq 2$, and since the system $\left\{\phi_{k}\right\}$ arises from a Birkhoff regular problem with simple spectrum, the theory of spectral operators cannot be used when $p \neq 2$.

Definition. Two bases $\left\{U_{k}\right\},\left\{\Phi_{k}\right\}$ of a Banach space $X$ are equivalent if there exists a bicontinuous linear map $A: X \rightarrow X$ such that $A \Phi_{k}=U_{k}$ for all $k$.

A theorem of Lorch [16] states that if $\left\{U_{k}\right\}$ and $\left\{\Phi_{k}\right\}$ are unconditional bases for a Hilbert space, and if $m^{-1} \leqslant\left\|U_{k}\right\|,\left\|\Phi_{k}\right\| \leqslant m$ for some $m>0$, then the two bases are equivalent. Conversely, it is clear that if two bases are equivalent, and if one is unconditional, then the other is unconditional. Thus the Dunford and Schwartz result is equivalent to showing that the system $\left\{u_{k}\right\}$ of root functions of a Birkhoff regular problem with simple spectrum is a basis in $L^{2}(0,1)$ equivalent to the trigonometric system $\left\{\phi_{k}\right\}$. It is this formulation which can be extended to all $L^{p}(0,1), 1<p<\infty$.

For any biorthogonal system having the properties stated in the Abstract, the expansion $\Sigma_{-\infty}^{\infty}\left(f, v_{k}\right) u_{k}$ converges unconditionally to $f$ in $L^{p}(0,1)$ if and only if $\Sigma_{-\infty}^{\infty}\left(A^{-1} f, \phi_{k}\right) \phi_{k}$ converges unconditionally to $f, 1<p<\infty$. In addition, the set of $L^{p}$ multiplier sequences for $\left\{u_{k}\right\}$ is the same as the set of multipliers for $\left\{\phi_{k}\right\}$.

The pointwise convergence of eigenfunction expansions was studied by $\mathbf{M}$. H. Stone [21], who showed that for any $f$ in $L^{1}(0,1)$, and for any Birkhoff regular problem,

$$
\sum_{-\infty}^{\infty}\left[\left(f, v_{k}\right) u_{k}(x)-\left(f, \phi_{k}\right) \phi_{k}(x)\right]=0
$$

uniformly on each interval $[\delta, 1-\delta], 0<\delta<1 / 2$. Combining this with the 
result of Carleson and Hunt on the almost everywhere convergence of Fourier series, we obtain the almost everywhere convergence of Birkhoff regular eigenfunction expansions. This paper provides a new proof of this for the case of simple spectrum.

Our result is similar to results in [10], [11] for real-valued orthonormal systems in $L^{p}(0,1), 1<p<\infty$, and to applications there to singular and nonsingular Sturm-Liouville systems. The asymptotic relation to the Fourier system is different there.

A slight change in point of view leads to the problem of perturbation of bases. As before, let $\left\{\Phi_{k}\right\}$ denote a basis for $X$, but now $\left\{U_{k}\right\}$ is just a sequence in $X$ such that $\Sigma a_{k} U_{k}=0$ is valid only when $a_{k}=0$ for all $k$. If, in some sense, $\left\{U_{k}\right\}$ is a sufficiently small perturbation of $\left\{\Phi_{k}\right\}$, and if $\left\{\Psi_{k}\right\}$ denotes the sequence in the dual space $X^{*}$ of functionals conjugate to $\left\{\Phi_{k}\right\}$, then $A f=\Sigma\left(f, \Psi_{k}\right) U_{k}$ defines a bicontinuous linear map on $X$. Since $A \Phi_{k}=$ $U_{k}$, we conclude that $\left\{U_{k}\right\}$ is a basis equivalent to $\left\{\Phi_{k}\right\}$. See [19] for a survey of results and further references.

There are a number of papers concerned with showing that more or less general classes of eigenfunction systems are bases by virtue of being perturbations of more familiar systems. See [6] for a prototype of the contraction operator method (in the language of [19]). See also [24, p. 86]. See [23] for the prototype of the compact operator method. More recent results can be found in [5, p. 337], [7] (in not quite the generality stated here) [15], [4].

In $\$ 2$ we prove an abstract theorem on perturbation of bases in Banach speces, and we introduce the terms needed to discuss almost everywhere convergence. In $\$ 3$ we show that certain subadditive (and possibly linear) mappings on $L^{p}(0,1)$ are of weak type $(p, p)$ (and possibly of strong type $(p, p))$. In $\S 4$ we define the asymptotic relations mentioned in the Abstract and prove the theorem on equivalence of bases and almost everywhere convergence. In $\$ 5$ we show that the root functions of Birkhoff regular problems with simple spectrum satisfy the asymptotic relations.

2. Transplantation theorems. Let $\left\{\Phi_{k}\right\}$ denote a basis for the Banach space $X$, with conjugate functionals $\left\{\Psi_{k}\right\}$ in $X^{*}$. Thus for each $f$ in $X$,

$$
f=\sum_{-\infty}^{\infty}\left(f, \Psi_{k}\right) \Phi_{k} .
$$

Let $\left\{U_{k}\right\}$ be another sequence in $X$, with complementary sequence $\left\{V_{k}\right\}$ in $X^{*}$ such that $\left(U_{k}, V_{j}\right)=\delta_{k j}$.

THEOREM 2.1. If $\left\{U_{k}\right\}$ is complete in $X$, then $\left\{U_{k}\right\}$ is a basis for $X$ equivalent to $\left\{\Phi_{k}\right\}$ if and only if

$$
A f=\sum_{-\infty}^{\infty}\left(f, \Psi_{k}\right) U_{k},
$$




$$
B f=\sum_{-\infty}^{\infty}\left(f, V_{k}\right) \Phi_{k}
$$

converge for all $f$ in $X$ and represent bounded linear operators.

Proof. Suppose $\left\{U_{k}\right\}$ is a basis for $X$ equivalent to $\left\{\Phi_{k}\right\}$. Let $A$ denote the bicontinuous map such that $A \Phi_{k}=U_{k}$. Then applying $A$ to (2.1), we see that the series in (2.2) represents a bounded linear operator. To get (2.3) we interchange the roles of $\left\{\Phi_{k}\right\}$ and $\left\{U_{k}\right\}$, and use $B=A^{-1}$.

For the converse, suppose the series (2.2), (2.3) represent bounded linear operators on $X$. Then $A \Phi_{k}=U_{k}, B U_{k}=\Phi_{k}$. Also, by (2.1), BA=I, and for each $f$ in $X$,

$$
A B f=\sum_{-\infty}^{\infty}\left(f, V_{k}\right) U_{k}
$$

is a bounded linear operator. It remains to show that $A B f=f$. Let $A B f=g$. Then $\left(g, V_{k}\right)=\left(f, V_{k}\right)$ for all $k$, and by the completeness of $\left\{U_{k}\right\}, g=f$.

The discussion of pointwise convergence requires concepts of mappings of weak type $(p, p)$, and the maximal function map. For fixed $p, p<\infty$, let $S$ denote a mapping (not necessarily linear) defined on $L^{p}(0,1)$, so that for $f$ in $L^{p}(0,1), S f$ is a function on $[0,1]$, not necessarily in $L^{p}(0,1)$. Let $m$ denote Lebesgue measure.

Definition. The mapping $S$ is of weak type $(p, p)$ if for each $f$ in $L^{p}(0,1)$, and each $y>0$,

$$
m\{x:|S f(x)|>y\} \leqslant K\left[\|f\|_{p} y^{-1}\right]^{p}
$$

where the constant $K>0$ does not depend on $f$ or $y$.

Let $\left\{u_{k}\right\}$ in $L^{p}(0,1)$ and $\left\{v_{k}\right\}$ in $L^{q}(0,1), p q=p+q$, be a biorthogonal system. For $f$ in $L^{p}(0,1)$, let

$$
S_{N}(x, f)=\sum_{-N}^{N}\left(f, v_{k}\right) u_{k}(x) .
$$

Definition. The mapping $f \rightarrow S^{*} f$ defined by

$$
\left(S^{*} f\right)(x)=\sup _{N}\left|S_{N}(x, f)\right|
$$

is called the maximal function mapping.

THEOREM $2.2[17$, p. 8]. The equation

$$
f(x)=\sum_{-\infty}^{\infty}\left(f, v_{k}\right) u_{k}(x), \text { a.e., }
$$

holds for all $f$ in $L^{p}(0,1)$ provided

(a) it holds for all $f$ in a dense subset of $L^{p}(0,1)$, and

(b) $S^{*}$ is of weak type $(p, p)$. 
3. Mappings on $L^{p}(0,1)$. Recall that $\phi_{k}(x)=\exp 2 k \pi i x$. For $f$ a function defined on $0 \leqslant x \leqslant 1$, consider the mappings $f \rightarrow g$ defined by

$$
\begin{aligned}
g(x) & =f(1-x), \\
g(x) & =X(x) f(x), \quad X \text { in } L^{\infty}(0,1), \\
g & =\sum_{0}^{\infty}\left(f, \phi_{k}\right) \phi_{k}, \\
g & =\sum_{-\infty}^{0}\left(f, \phi_{k}\right) \phi_{k}, \\
g & =\sum_{-\infty}^{\infty} a_{k}\left(f, \phi_{k}\right) \phi_{k}, \quad a_{k}=O\left(|k|^{-1 / 2}\right) .
\end{aligned}
$$

These are all bounded linear mappings on $L^{p}(0,1), 1<p<\infty$. The first two are obvious. For (3.3) and (3.4) we use the fact that the spaces $L^{p}(0,1)$ admit conjugation for $1<p<\infty[14$, p. 48]. For (3.5) we use the fact that any sequence $a_{k}=O\left(|k|^{-1 / 2}\right)$ is a multiplier sequence for $L^{p}(0,1), 1<p<\infty$ $[13$, p. 231].

Let $e_{k}(x)=\exp [-2 k \pi(\alpha+i \beta) x], \quad e_{k}^{\prime}(x)=\exp [-2 k \pi(\gamma+i \delta) x], \quad k=$ $0,1, \ldots$ Consider the mapping

$$
g=\sum_{0}^{\infty}\left(f, e_{k}^{\prime}\right) e_{k}
$$

If $\alpha=\gamma=0$, then with appropriate rescaling of the variable this is of the form (3.3). If $f$ is in $L^{2}(0,1)$ and if $\gamma \geqslant 0$, then $\left\{\left(f, e_{k}^{\prime}\right)\right\}$ is in $l^{2}$ [9, p. 2332]. Thus $\Sigma_{0}^{\infty}\left(f, e_{k}^{\prime}\right) \phi_{k}$ represents a bounded linear operator on $L^{2}(0,1)$. Its adjoint is $\Sigma_{0}^{\infty}\left(f, \phi_{k}\right) e_{k}^{\prime}$, which is then also a bounded linear operator on $L^{2}(0,1)$. Finally then we see that (3.6) is a bounded linear operator on $L^{2}(0,1)$ if $\alpha \geqslant 0$, $\gamma>0$.

THEOREM 3.1. If $\alpha \geqslant 0, \gamma \geqslant 0,|\beta| \leqslant 1$, then (3.6) is a bounded linear operator on $L^{p}(0,1), 1<p \leqslant 2$.

Proof. The case $\alpha=\gamma=0$ is contained in (3.3), so we consider the case that $\alpha+\gamma>0$. We shall show that (3.6) is of weak type $(1,1)$. Since we have just seen that $(3.6)$ is of strong type $(2,2)$, we can apply the Marcinkiewicz interpolation theorem $[25$, p. 112].

Let $f$ be in $L^{1}(0,1)$, and let $g_{N}(X)=\Sigma_{0}^{N-1}\left(f, e_{k}^{\prime}\right) e_{k}(x)$. Then 


$$
\begin{aligned}
g_{N}(x)= & \int_{0}^{1} \sum_{0}^{N-1} e^{-2 k \pi[(\alpha x+\gamma t)+i(\beta x-\delta t)]} f(t) d t \\
= & \int_{0}^{1}\left[1-e^{-2 \pi[(\alpha x+\gamma t)+i(\beta x-\delta t)]}\right]^{-1} f(t) d t \\
& -e^{-2 N \pi(\alpha+i \beta) x} \int_{0}^{1}\left[1-e^{-2 \pi[(\alpha x+\gamma t)+i(\beta x-\delta t)]}\right]^{-1} e^{-2 N \pi(\gamma-i \delta) t} f(t) d t,
\end{aligned}
$$

provided $1-e^{-2 \pi[(\alpha x+\gamma t)+i(\beta x-\delta t)]} \neq 0$. If $0<x<1$ and $|\beta| \leqslant 1$, this is the case. If at least one of $\alpha, \gamma$ is not zero, the last term in (3.7) converges to zero as $N \rightarrow \infty$, for $0<x<1$. Thus

$$
g(x)=\int_{0}^{1}\left[1-e^{-2 \pi[(\alpha x+\gamma t)+i(\beta x-\delta t)]}\right]^{-1} f(t) d t .
$$

Now there exists a constant $K^{-1}>0$ such that for all points in the spiral $\exp [-2 \pi[(\alpha x+\gamma t)+i(\beta x-\delta t)]]$, we have

$$
\left|1-e^{-2 \pi[(\alpha x+\gamma t)+i(\beta x-\delta t)]}\right| \geqslant K^{-1} x(1-x) .
$$

Thus

$$
|g(x)| \leqslant K\|f\|_{1} x^{-1}(1-x)^{-1}, \quad 0<x<1 .
$$

This implies that

$$
m\{x:|g(x)|>y\} \leqslant K\|f\| y^{-1}, \quad y>0,
$$

so the map $f \rightarrow g$ is of weak type $(1,1)$.

THEOREM 3.2. If $f$ is in $L^{p}(0,1), 1 \leqslant p \leqslant 2$, then the map $f \rightarrow\left\{\left(f, e_{k}\right)\right\}$ is a bounded linear operation into $l^{q}, p q=p+q$.

Proof. For $p=2$ this is proved in $[9$, p. 2332]. For $p=1$ the result is obvious. Then the Riesz-Thorin theorem [25, p. 95] gives the result for the remaining values of $p$.

Note that the boundedness of this map yields

$$
\left[\sum_{0}^{\infty}\left|\left(f, e_{k}\right)\right|^{q}\right]^{1 / q} \leqslant K\|f\|_{p}, \quad 1<p \leqslant 2 .
$$

Let $g^{*}(x)=\sup _{N}\left|g_{N}(x)\right|$.

THEOREM 3.3. The mapping $f \rightarrow g^{*}$ is of weak type $(p, p)$ for $1 \leqslant p \leqslant 2$ if $\alpha>0$.

Proof. The case that $p=1$ is contained in the proof of Theorem 3.1. For $1<p \leqslant 2$ we use (3.8) and Hölder's inequality: 


$$
\begin{aligned}
\left|g_{N}(x)\right| & \leqslant\left[\sum_{0}^{N-1}\left|\left(f, e_{k}^{\prime}\right)\right|^{q}\right]^{1 / q}\left[\sum_{0}^{N-1}\left|e_{k}(x)\right|^{p}\right]^{1 / p} \\
& \leqslant K\|f\|_{p}\left[\sum_{0}^{\infty} e^{-2 \pi \alpha p x k}\right]^{1 / p}=K\|f\|_{p}\left[1-e^{-2 \pi \alpha p x}\right]^{-1 / p} \\
& \leqslant K^{\prime}\|f\|_{p} x^{-1 / p}, \quad 0<x \leqslant 1 .
\end{aligned}
$$

Thus $g^{*}(x) \leqslant K^{\prime}\|f\|_{p} x^{-1 / p}$. Thus $m\left\{x: g^{*}(x)>y\right\} \leqslant K\left[\|f\|_{p} y^{-1}\right]^{p}$.

4. Main theorems. Let $\langle 1\rangle$ denote any function of the form

$$
1+a_{k 1} x|k|^{-1 / 2}+a_{k 2} x^{2} k^{-1}+O\left(k^{-3 / 2}\right),
$$

uniformly in $x, 0 \leqslant x \leqslant 1$, as $|k| \rightarrow \infty$, and $\left\{a_{k i}\right\}$ is in $l^{\infty}, i=1,2$. Let $F_{k}(x)$ denote any finite linear combination of functions of the form

$$
\left[A+O\left(k^{-1}\right)\right] e_{|k|}(x), \quad\left[A+O\left(k^{-1}\right)\right] e_{|k|}(1-x)
$$

where $O\left(k^{-1}\right)$ is uniform in $x, 0 \leqslant x \leqslant 1$ as $|k| \rightarrow \infty$ and $e_{|k|}$ is generic notation for any function $\exp [-2|k| \pi(\alpha+i \beta) x]$, with $\alpha>0,|\beta| \leqslant 1$. $A$ is any constant.

TheOREM 4.1. Let $p$ be fixed, $1<p<\infty$. Let $\left\{u_{k}(x)\right\}$ in $L^{p}(0,1)$, and $\left\{v_{k}(x)\right\}$ in $L^{q}(0,1), k=0, \pm 1, \ldots$, be complete sequences which are biorthogonal: $\left(u_{k}, v_{j}\right)=\delta_{k j}$. Suppose also that

$$
\begin{aligned}
& u_{k}(x)=X_{1}(x) \phi_{k}(x)\langle 1\rangle+X_{2}(x) \phi_{-k}(x)\langle 1\rangle+F_{k}(x), \quad k \rightarrow+\infty, \\
& v_{k}(x)=Y_{1}(x) \phi_{k}(x)\langle 1\rangle+Y_{2}(x) \phi_{-k}(x)\langle 1\rangle+F_{k}(x), \quad k \rightarrow+\infty \text {, } \\
& u_{k}(x)=X_{3}(x) \phi_{k}(x)\langle 1\rangle+X_{4}(x) \phi_{-k}(x)\langle 1\rangle+F_{k}(x), \quad k \rightarrow-\infty \text {, } \\
& v_{k}(x)=Y_{3}(x) \phi_{k}(x)\langle 1\rangle+Y_{4}(x) \phi_{-k}(x)\langle 1\rangle+F_{k}(x), \quad k \rightarrow-\infty,
\end{aligned}
$$

where $X_{i}, Y_{i}$ are in $L^{\infty}(0,1), i=1, \ldots, 4$. Then $\left\{u_{k}\right\}$ is a basis for $L^{p}(0,1)$ equivalent to $\left\{\phi_{k}\right\}$, and $\left\{v_{k}\right\}$ is a basis for $L^{q}(0,1)$ equivalent to $\left\{\phi_{k}\right\}$.

Proof. Since the hypotheses are symmetric with respect to the two sequences $\left\{u_{k}\right\},\left\{v_{k}\right\}$, there is no loss of generality in assuming $1<p \leqslant 2$. Applying Theorem 2.1, consider

$$
\begin{aligned}
A f= & \sum_{-\infty}^{\infty}\left(f, \phi_{k}\right) u_{k}=X_{1}(x) \sum_{0}^{\infty}\left(f, \phi_{k}\right) \phi_{k}\langle 1\rangle+X_{2}(x) \sum_{0}^{\infty}\left(f, \phi_{k}\right) \phi_{-k}\langle 1\rangle \\
& +X_{3}(x) \sum_{-\infty}^{-1}\left(f, \phi_{k}\right) \phi_{k}\langle 1\rangle+X_{4}(x) \sum_{-\infty}^{-1}\left(f, \phi_{k}\right) \phi_{-k}\langle 1\rangle+\sum_{-\infty}^{\infty}\left(f, \phi_{k}\right) F_{k} .
\end{aligned}
$$

The first term on the right of (4.1) becomes

$$
X_{1}(x)\left\{\sum_{0}^{\infty}\left(f, \phi_{k}\right) \phi_{k}+\sum_{0}^{\infty}\left(f, \phi_{k}\right) a_{k 1} k^{-1 / 2} \phi_{k}+\sum_{0}^{\infty}\left(f, \phi_{k}\right) O\left(k^{-1}\right) \phi_{k}\right\} \text {. }
$$


The first two terms in the bracket represent bounded linear operators, by virtue of (3.3), (3.5) respectively. For the third term, we use the HausdorffYoung inequality and Hölder's inequality:

$$
\begin{aligned}
\left|\sum_{0}^{\infty}\left(f, \phi_{k}\right) O\left(k^{-1}\right) \phi_{k}\right| & \leqslant\left[\sum_{0}^{\infty}\left|\left(f, \phi_{k}\right)\right|^{q}\right]^{1 / q}\left(\sum_{1}^{\infty} k^{-p}\right)^{1 / p} \\
& \leqslant M\|f\|_{p} .
\end{aligned}
$$

Finally, multiplication by $X_{1}$ is a bounded operation.

For the second term on the right of (4.1), we see that if $F(x)=f(1-x)$, then $\left(F, \phi_{-k}\right)=\left(f, \phi_{k}\right)$, so the second term can be written as

$$
X_{2} \sum_{0}^{\infty}\left(F, \phi_{-k}\right) \phi_{-k}\langle 1\rangle
$$

and then treated exactly as the first term. These arguments hold also for the third and fourth terms in (4.1). For the fifth term, we note that a typical expression is

$$
\sum_{0}^{\infty}\left(f, \phi_{k}\right)\left[1+O\left(k^{-1}\right)\right] e_{k}=\sum_{0}^{\infty}\left(f, \phi_{k}\right) e_{k}+\sum_{0}^{\infty}\left(f, \phi_{k}\right) O\left(k^{-1}\right) e_{k} .
$$

The first term on the right is bounded by virtue of Theorem 3.1. For the second term we again use the Hausdorff-Young inequality. Thus $A$ is a bounded linear operator.

Next we consider

$$
\begin{aligned}
B f= & \sum_{-\infty}^{\infty}\left(f, v_{k}\right) \phi_{k}=\sum_{0}^{\infty}\left(f, Y_{1} \phi_{k}\langle 1\rangle\right) \phi_{k}+\sum_{0}^{\infty}\left(f, Y_{2} \phi_{-k}\langle 1\rangle\right) \phi_{k} \\
& +\sum_{-\infty}^{-1}\left(f, Y_{3} \phi_{k}\langle 1\rangle\right) \phi_{k}+\sum_{-\infty}^{-1}\left(f, Y_{4} \phi_{-k}\langle 1\rangle\right) \phi_{k}+\sum_{-\infty}^{\infty}\left(f, F_{k}\right) \phi_{k} .
\end{aligned}
$$

The first term on the right of (4.2) becomes

$$
\begin{aligned}
& \sum_{0}^{\infty}\left(\bar{Y}_{1} f, \phi_{k}\right) \phi_{k}+\sum_{0}^{\infty}\left(x \bar{Y}_{1} f, \phi_{k}\right) a_{k 1} k^{-1 / 2} \phi_{k} \\
& +\sum_{0}^{\infty}\left(x^{2} \bar{Y}_{1} f, \phi_{k}\right) a_{k 2} k^{-1} \phi_{k}+\sum_{0}^{\infty}\left(\bar{Y}_{1} f, \phi_{k} O\left(k^{-3 / 2}\right)\right) \phi_{k} .
\end{aligned}
$$

These terms clearly represent bounded linear operators, and thus the first four terms on the right of (4.2) are bounded. For the fifth term, a typical expression is

$$
\sum_{0}^{\infty}\left(f,\left[1+O\left(k^{-1}\right)\right] e_{k}\right) \phi_{k}=\sum_{0}^{\infty}\left(f, e_{k}\right) \phi_{k}+\sum_{0}^{\infty}\left(f, O\left(k^{-1}\right) e_{k}\right) \phi_{k} .
$$


The first term is bounded by virtue of Theorem 3.1. For the second term, we use Theorem 3.2 and Hölder's inequality:

$$
\left|\sum_{1}^{\infty}\left(f, e_{k}\right) k^{-1}\right| \leqslant\left[\sum_{1}^{\infty}\left|\left(f, e_{k}\right)\right|^{q}\right]^{1 / q}\left(\sum_{1}^{\infty} k^{-p}\right)^{1 / p}=m\|f\|_{p} .
$$

Thus $B$ is also a bounded linear operator.

Since $A \phi_{k}=u_{k}$ implies $A^{-1} * \phi_{k}=v_{k}$, we have

$$
\left(A f, v_{k}\right)=\left(f, \phi_{k}\right) \text {. }
$$

THEOREM 4.2. Let $\left\{u_{k}\right\},\left\{v_{k}\right\}$ be as in Theorem 4.1. Let $f$ be in $L^{p}(0,1)$, $1<p$. Then $f(x)=\Sigma_{-\infty}^{\infty}\left(f, v_{k}\right) u_{k}(x)$, a.e.

PRoof. We apply Theorem 2.2. Let $\Delta$ denote the class of functions $f$ of class $C^{2}(0,1)$, such that $f$ and $f^{(1)}$ are zero at $x=0$ and $x=1$. For such $f$, $\left(f, \phi_{k}\right)=O\left(k^{-2}\right)$, and thus the Fourier series of $f$ converges uniformly to $f$ on $[0,1]$. Clearly $\Delta$ is dense in $L^{p}(0,1), p<\infty$, and since $A$ is continuous, $A \Delta$ is dense in $L^{p}(0,1)$. Using (4.3), we see that if $g=A f$, then $\left(g, v_{k}\right)=O\left(k^{-2}\right)$. Since the $u_{k}$ 's are uniformly bounded, we see that for $g$ in $A \Delta$, $\sum_{-\infty}^{\infty}\left(g, v_{k}\right) u_{k}(x)$ converges uniformly on $[0,1]$ to $g(x)$. This establishes condition (a) of Theorem 2.2.

To establish condition (b), we note first of all that the maximal function mapping for ordinary Fourier series is of weak type $(p, p)$ (in fact, of strong type $(p, p))[17$, p. 8$]$. Now

$$
\begin{aligned}
S_{N}(x, f)= & \sum_{-N}^{N}\left(f, v_{k}\right) u_{k}(x) \\
= & X_{1}(x) \sum_{0}^{N}\left(\bar{Y}_{1} f, \phi_{k}\langle 1\rangle\right) \phi_{k}(x)\langle 1\rangle \\
& +X_{1}(x) \sum_{0}^{N}\left(\bar{Y}_{2} f, \phi_{-k}\langle 1\rangle\right) \phi_{k}(x)\langle 1\rangle \\
& +X_{2}(x) \sum_{0}^{N}\left(\bar{Y}_{1} f, \phi_{k}\langle 1\rangle\right) \phi_{-k}(x)\langle 1\rangle \\
& +X_{2}(x) \sum_{0}^{N}\left(\bar{Y}_{2} f, \phi_{-k}\langle 1\rangle\right) \phi_{-k}(x)\langle 1\rangle \\
& +X_{1}(x) \sum_{0}^{N}\left(f, F_{k}\right) \phi_{k}(x)\langle 1\rangle+X_{2}(x) \sum_{0}^{N}\left(f, F_{k}\right) \phi_{-k}(x)\langle 1\rangle \\
& +\sum_{0}^{N}\left(\bar{Y}_{1} f, \phi_{k}\langle 1\rangle\right) F_{k}(x)+\sum_{0}^{N}\left(\bar{Y}_{2} f, \phi_{-k}\langle 1\rangle\right) F_{k}(x) \\
& +\sum_{0}^{N}\left(f, F_{k}\right) F_{k}(x)+\sum_{-N}^{-1}\left(f, v_{k}\right) u_{k}(x) .
\end{aligned}
$$


The first four terms on the right of (4.4) can each be further decomposed, into the partial sums of ordinary Fourier series. Thus each of these terms yields maximal functions of weak type $(p, p)$. The fifth through ninth terms can be handled using Theorem 3.3. The last term is handled in a similar way. Note that there is no loss of generality in assuming $1<p \leqslant 2$.

5. Eigenvalues and eigenfunctions of differential operators. Let $(\tau, U)$ denote an $n$th order Birkhoff regular boundary value problem with simple spectrum. Then the eigenfunctions $\left\{u_{k}\right\}$ satisfy the conditions of Theorem 4.1, and the eigenfunctions $\left\{v_{k}\right\}$ of the adjoint problem also satisfy these conditions. To prove this, we need to know the asymptotic distribution of the eigenvalues of $(\tau, U)$, and we need to know how to form the root functions of $(\tau, U)$ from a fundamental set of solutions to $\tau u=\lambda u$.

If $\lambda$ is the eigenvalue parameter, it is customary to introduce a new parameter by $\lambda=-\rho^{n}$, and then restrict attention to a sector of the $\rho$-plane of opening $2 \pi / n$. $S_{l}$ denotes the sector $l \pi / n \leqslant \arg \rho \leqslant(l+1) \pi / n$. The symbols $\theta_{-1}, \theta_{0}, \theta_{1}$ are defined in [18, p. 49], and the $n$th roots of -1 ; $\omega_{1}, \ldots, \omega_{n}$ are labeled as in [18, p. 49]. If $\lambda$ is an eigenvalue of $(\tau, U)$, we shall commonly refer to $\rho$ as an eigenvalue, where it will always be clear which root of $\lambda$ is meant.

The asymptotic behavior of the eigenvalues of a Birkhoff regular $(\tau, U)$ is completely described in [18]. We restate the result in terms of $\rho$ rather than $\lambda$.

THEOREM $5.1[18$, p. 56]. Let $(\tau, U)$ denote an nth order Birkhoff regular boundary value problem.

I. If $n=2 \mu-1$, then all but finitely many eigenvalues are of algebraic multiplicity one, and in each sector $S_{l}, l=0,1$, there is a sequence of eigenvalues satisfying

$$
\rho_{k}= \pm 2 k \pi i \omega_{\mu}^{-1}+A+O\left(k^{-1}\right), \quad k \rightarrow+\infty,
$$

where $A$ is a constant depending on $l$, and the choice of sign depends upon $l$ and the value of $n$ modulo 4.

II. If $n=2 \mu$ and $\theta_{0}^{2}-4 \theta_{-1} \theta_{1} \neq 0$, then all but finitely many eigenvalues of $(\tau, U)$ are of algebraic multiplicity one, and $S_{0}$ contains two sequences of eigenvalues, each satisfying

$$
\rho_{k}= \pm 2 k \pi i \omega_{\mu}^{-1}+A+O\left(k^{-1}\right), \quad k \rightarrow \infty,
$$

where the constant $A$ is not the same for both sequences, and the choice of sign depends upon the value of $n$ modulo 4.

The asymptotic behavior of eigenfunctions is also discussed in [18]. Let [ $B$ ] denote any function of the form $B+O\left(k^{-1}\right)$ as $|k| \rightarrow \infty$, uniformly in $x$, $0 \leqslant x \leqslant 1$. 
THEOREM 5.2 [18, pp. 65-68]. Let $(\tau, U)$ denote an nth order Birkhoff regular boundary value problem.

I. If $n=2 \mu-1$ and $\left|\rho_{k}\right|$ is sufficiently large, then the corresponding eigenfunction $u_{k}(x)$ is given by

$$
\begin{gathered}
u_{k}(x)=\left[B_{\mu}\right] \exp \left(\rho_{k} \omega_{\mu} x\right)+\sum_{l=1}^{\mu-1}\left[B_{l}\right] \exp \left(\rho_{k} \omega_{l} x\right) \\
+\sum_{l=\mu+1}^{n}\left[B_{l}\right] \exp \left(\rho_{k} \omega_{l}(x-1)\right) ;
\end{gathered}
$$

where $B_{\mu} \neq 0$.

II. If $n=2 \mu, \theta_{0}^{2}-4 \theta_{-1} \theta_{1} \neq 0$ and $\left|\rho_{k}\right|$ is sufficiently large, then the corresponding eigenfunction $u_{k}(x)$ is given by

$$
\begin{aligned}
u_{k}(x)=[ & \left.B_{\mu}\right] \exp \left(\rho_{k} \omega_{\mu} x\right)+\left[B_{\mu+1}\right] \exp \left(\rho_{k} \omega_{\mu+1} x\right) \\
& +\sum_{l=1}^{\mu-1}\left[B_{l}\right] \exp \left(\rho_{k} \omega_{l} x\right)+\sum_{l=\mu+2}^{n}\left[B_{l}\right] \exp \left(\rho_{k} \omega_{l}(x-1)\right),
\end{aligned}
$$

where $\left|B_{\mu}\right|+\left|B_{\mu+1}\right|>0$.

Note that the constants $B_{\mu}, B_{\mu+1}$ are not zero as a consequence of Birkhoff regularity.

THEOREM 5.3. If $(\tau, U)$ is any Birkhoff regular problem with simple spectrum, then the eigenfunctions $\left\{u_{k}\right\}$ satisfy the conditions of Theorem 4.1.

Proof. In case I, consider (5.1) and (5.3). We have for $k \rightarrow \infty$,

$$
\exp \left(\rho_{k} \omega_{\mu} x\right)=\exp (2 k \pi i x) \exp A x\langle 1\rangle
$$

so

$$
\left[B_{\mu}\right] \exp \left(\rho_{k} \omega_{\mu} x\right)=B_{\mu} \exp A x \phi_{k}(x)\langle 1\rangle .
$$

The other terms in (5.3) contribute to $F_{k}(x)$. For $k \rightarrow-\infty$ we get different $B_{\mu}$ and $A$. Thus when $n$ is odd, $X_{2}$ and $X_{4}$ are identically zero. Case II is handled similarly, using (5.2), (5.3), and the relation $\theta_{0}^{2}-4 \theta_{1} \theta_{-1} \neq 0$.

It is necessary to obtain similar estimates for the eigenfunctions $\left\{v_{k}\right\}$ of the adjoint problem. Since the coefficients of $\tau$ are not necessarily differentiable, the adjoint problem is not necessarily a differential operator. However, the explicit form of the adjoint is not of importance. Since $(\tau, U)$ generates a densely defined closed linear operator in each space $L^{p}(0,1), 1<p<\infty$, we know from abstract considerations [12, p. 43] that an adjoint problem exists as a densely defined operator in $L^{q}(0,1), p q=p+q$. The eigenfunctions of the adjoint problem can be obtained from the Green's function $G(x, t, \rho)$ of $(\tau, U)$. If $\rho_{k}$ is an eigenvalue of $(\tau, U)$, then for fixed $x, t, n \rho^{n-1} G(x, t, \rho)$ has a 
pole at $\rho_{k}$. If the algebraic and geometric multiplicity of $\rho_{k}$ are both one, then the principal part of $n \rho^{n-1} G(x, t, \rho)$ at $\rho_{k}$ is

$$
u_{k}(x) \bar{v}_{k}(t)\left[\rho-\rho_{k}\right]^{-1} \text {. }
$$

If the algebraic and geometric multiplicities are both two, then the principal part is

$$
\left[u_{k}^{(0)}(x) \bar{v}_{k}^{(0)}(t)+u_{k}^{(1)}(x) \bar{v}_{k}^{(1)}(t)\right]\left[\rho-\rho_{k}\right]^{-1},
$$

where $u_{k}^{(0)}, u_{k}^{(1)}$ are two linearly independent eigenfunctions.

Consider the case that $n$ is odd, so (5.5) is applicable. Then

$$
u_{k}(x) \bar{v}_{k}(t)=\lim _{\rho \rightarrow \rho_{k}}\left(\rho-\rho_{k}\right) n \rho^{n-1} G(x, t, \rho) .
$$

Suppose $k$ is large. Then

$$
\int_{0}^{1} u_{k}(x)\left[\bar{B}_{\mu}^{-1} e^{-\bar{A} x} \phi_{k}(x)\right]^{-} d x=\langle 1\rangle
$$

so

$$
\langle 1\rangle \bar{v}_{k}(t)=\int_{0}^{1}\left[\lim _{\rho \rightarrow \rho_{k}}\left(\rho-\rho_{k}\right) n \rho^{n-1} G(x, t, \rho)\right]\left[\bar{B}_{\mu}^{-1} e^{-\overline{A x}} \phi_{k}(x)\right]^{-} d x .
$$

Now the integral in (5.6) can be evaluated using equation (4.1) and Lemma 4.1 , both in [1]. (In the last line of the statement of Lemma 4.1, replace $\omega_{i}$ by $\omega_{j}$.) Labeling the $\omega_{i}$ 's according to [18] rather than [1], we see that

$$
\lim _{\rho \rightarrow \rho_{k}}\left(\rho-\rho_{k}\right) p_{\mu \mu}(\rho)=C_{\mu}+O\left(k^{-1}\right), \quad C_{\mu} \neq 0,
$$

while the residues of the other $p_{k j}(\rho)$ 's reflect the exponential decay of the $F_{k}$ 's. Thus the right side of (5.6) is

$$
C_{\mu} e^{D x} \phi_{k}(x)\langle 1\rangle+F_{k}(x)
$$

and then $\bar{v}_{k}(t)$ has the same form. The case that $n$ is even is handled in a similar manner.

THEOREM 5.4. Let $(\tau, U)$ denote an nth order Birkhoff regular problem with simple spectrum. Then the eigenfunctions $\left\{v_{k}\right\}$ of the adjoint problem satisfy the conditions of Theorem 4.1.

REMARK. There are Birkhoff regular problems whose eigenvalues have algebraic multiplicity equal to two and geometric multiplicity equal to one. The root functions of such problems are a basis for each $L^{p}(0,1)[3]$, but in general such a basis is conditional even if $p=2$ [22].

\section{REFERENCES}

1. H. E. Benzinger, Green's function for ordinary differential operators, J. Differential Equations 7 (1970), 478-496. MR 41 \#189. 
2. Completeness of eigenvectors in Banach spaces, Proc. Amer. Math. Soc. 38 (1973), 319-324. MR 47 \# 7487.

3. $\_$The $L^{p}$ behavior of eigenfunction expansions, Trans. Amer. Math. Soc. 174 (1972), 333-344. MR 48 \#6531.

4. _ـ An application of the Hausforff-Young inequality to eigenfunction expansions, Ordinary and Partial Differential Equations, Lecture Notes in Math., vol. 415, Springer-Verlag, Berlin and New York, 1974.

5. G. Birkhoff and G.-C. Rota, Ordinary differential equations. Introduction to higher mathematics, Ginn, Boston, Mass., 1963. MR 25 \#2253.

6. G. D. Birkhoff, $A$ theorem on series of orthogonal functions with an application to SturmLiouville series, Proc. Nat. Acad. Sci. U. S. A. 3 (1917), 656-659.

7. F. Brauer, On the completeness of biorthogonal systems, Michigan Math. J. 11 (1964), 379-383; Correction, ibid. 12 (1965), 127-128. MR 29 \#6095.

8. M. M. Day, Normed linear spaces, second printing corrected, Springer-Verlag, Berlin, 1962. MR 20 \#1187.

9. N. Dunford and J. T. Schwartz, Linear operators, III, Interscience, New York, 1971.

10. J. E. Gilbert, Maximal theorems for some orthogonal series. I, Trans. Amer. Math. Soc. 145 (1969), 495-515. MR 40 \#6156.

11. __ Maximal theorems for some orthogonal series. II, J. Math. Anal. Appl. 31 (1970), 349-368.

12. E. Hille and R. S. Phillips, Functional analysis and semi-groups, Amer. Math. Soc. Colloq. Publ., vol. 31, Amer. Math. Soc., Providence, R. I., 1957. MR 19, 664.

13. I. I. Hirschman, Jr., On multiplier transforms, Duke Math. J. 26 (1959), 221-242. MR 21 \#3721.

14. Y. Katznelson, An introduction to harmonic analysis, Wiley, New York, 1968. MR 40 \#1734.

15. J. L. Kazdan, Perturbation of complete orthonormal sets and eigenfunction expansions, Proc. Amer. Math. Soc. 27 (1971), 506-510. MR 42 \#6648.

16. E. R. Lorch, Bicontinuous linear transformations in certain vector spaces, Bull. Amer. Math. Soc. 45 (1939), 564-569. MR 1, 58.

17. C. J. Mozzochi, On the pointwise convergence of Fourier series, Lecture Notes in Math., vol. 199, Springer-Verlag, New York, 1971.

18. M. A. Neumark, Lineare Differentialoperatoren, Akademie-Verlag, Berlin, 1960; English transl., Ungar, New York, 1968. MR 41 \# 7485.

19. J. R. Retherford and J. R. Holub, The stability of bases in Banach and Hilbert spaces, J. Reine Angew. Math. 246 (1971), 136-146. MR 45 \#867.

20. I. Singer, Bases in Banach spaces. I, Springer-Verlag, Berlin, 1970. MR 45 \#7451.

21. M. H. Stone, A comparison of the series of Fourier and Birkhoff, Trans. Amer. Math. Soc. 28 (1926), 695-761.

22. P. W. Walker, Certain second order boundary value problems, Notices Amer. Math. Soc. 22 (1975), A-139. Abstract \#720-34-27.

23. J. L. Walsh, On the convergence of Sturm-Liouville series, Ann. of Math. (2) 24 (1921), 109-120.

24. N. Wiener and R. E. A. C. Paley, Fourier transforms in the complex domain, Amer. Math. Soc. Colloq. Publ., vol. 19, Amer. Math. Soc., Providence, R. I., 1934.

25. A. Zygmund, Trigonometric series, 2nd ed., vol. II, Cambridge Univ. Press, Cambridge, 1959. MR 21 \#6498.

Department of Mathematics, University of IlLinois, Urbana, IlLinoIs 61801 